Differential adaptation to gratings blocked by

\section{cubes and gratings blocked by hexagons: A test of the neural symbolic activity hypothesis*}

\author{
NAOMI WEISSTEIN, FANNY MONTALVO, and GREGORY OZOG \\ Loyola University, Chicago, Ill. 60626
}

After an $\mathbf{S}$ views a grating which is partially blocked from view, adaptation (reduction in apparent contrast) occurs to a subsequent grating presented within a portion of the visual field where the prior grating was visible and also within a portion where the grating had been blocked from view. This adaptation effect is statistically significant when a cube blocks the grating portion and is not statistically significant when an outline hexagon blocks the grating portion. This increase in adaptation with depth cues implies that such an effect is not due only to "simple spatial spread" or to eye movements.

The ability to separate a scene into its components is vital for any nontrivial pattern recognition. Computer pattern recognition simulations that can accomplish such separations make extensive use of internal models (Guzman, 1969). The simulations proceed in two steps which we shall call Step $a$ and Step b. In Step a, features are abstracted from the visual array; in Step b, relationships between these features are tested.

It has been suggested that human pattern recognition may proceed in a similar manner. There certainly is accumulating evidence for human visual system processes analogous to Step a. For instance, when a grating is viewed for a prolonged duration, thresholds for the same and similar gratings are subsequently raised or the apparent contrast of the same and similar gratings is reduced, while these effects do not occur for gratings differing in orientation or size (Blak emore \& Campbell, 1969). This is called adaptation and indicates that different populations of neurons respond differentially to features of a stimulus.

However, there is only scanty evidence for the existence of mechanisms similar to Step $b$. In one attempt at testing a Step b type mechanism, Weisstein (1970a) had Ss view a grating which was partially blocked from view by a perspective drawing of a cube. She found that adaptation occurred not only to those portions of the grating which were visible, but also to those portions blocked from view. She suggested that this effect could be interpreted in

*Supported by Grant 8 R01 EY 00143-05 from the National Eye Institute. terms of neural representation in the visual system of the concept "in back of." In other words, in terms of neural mechanisms, a relatively simple procedure for the separation of a scene into its components might be the firing, in response to those places in the visual field where an object was blocked from view, of a subset of those size and orientation selective neurons comprising the population which would ordinarily fire if the object were completely visible. If this subset were firing, it should adapt, just as the populations which fire directly to an object adapt.

There are, of course, a number of alternate explanations (Sekuler \& Armstrong, 1970). The effect might have been due to direct stimulation, i.e., eye movements may have been sufficiently large so that the same retinal area was stimulated both by the grating portion of the field and by the test grating. While this is highly unlikely (see Weisstein, 1970b, and below), an eye movement hypothesis can be partially tested by increasing the separation between the test and grating portion of the interrupted-grating stimulus and by adding a condition where a grating is simply blocked rather than blocked by a perspective drawing of an object which appears in front of it. (The only sufficient test of an eye movement $\mathrm{hypothesis} \mathrm{is} \mathrm{measuring} \mathrm{eye}$ movements, since, because the control and experimental figures which interrupt the grating are different, one can always hypothesize that each figure produces differential amounts of eye movements).

Sekuler \& Armstrong (1970) also suggested that the effect might be due to "simple spatial spread of the orientation-specific adaptation." While in no case would such a spatial spread be simple (see Weisstein, 1970b), their suggestion can be rephrased in terms of the distinction between Step a and Step b mechanisms. That is, the effect might result simply from antagonistic receptive field organization such as is associated with step a (feature extraction) mechanisms. In other words, portions of the outside adaptation grating might have inhibited portions of the subsequent test grating. The angular separations between adaptation and test gratings in Weisstein (1970a) were larger than would be expected for this hypothesis to be likely; however, this, too, can be tested by a condition where a grating is simply blocked.

Thus, gratings interrupted by a figure with (cube) and without (hexagon) depth cues may decide between a neural representation hypothesis and explanations which do not require such a hypothesis.

\section{METHOD}

This study replicated Weisstein's experiment, photographically enlarging the slides used in that experiment. This meant that there was a larger separation between test and adaptation stimuli (and, also, a lower spatial frequency of grating portions of the stimuli). Hexagon control conditions were added. Figure 1 shows the stimuli, transilluminated Kodalith slides, presented in a six-channel binocular tachistoscope (Scientific Prototype, Model GB). The first two rows are adaptation slides; the last row is the test slide (T). The gratings and blank are general controls, testing for adaptation to the grating and to the mean spatial luminance of the fields. The hexagon grating is one specific control for the cube grating; the cube and hexagon are additional controls in case the presence of figures in a blank field changes the blank field adaptation function.

Relative to the cube, $T$ was positioned in the center of the top plane. The minimum distance from the edge of the cube or hexagon to the circumference of $T$ as measured along the perpendicular to the hexagonal perimeter which passes through the center of $T$ was no less than 31 min of visual angle. An upper bound for the standard deviation of a fixating eye about its mean position is roughly 5 min (Steinman, 1965; Weisstein, $1970 \mathrm{~b})$. Thus, for the meridian along which there is maximum chance of adaptation due to eye movements, the probability of occurrence of eye movements large enough to produce such adaptation is extremely small.

All grating portions of the stimuli were $2.13-c /$ deg square waves with a contrast near 1 and a mean spatial luminance of $25 \mathrm{fL}$. The non-grating portions of the fields had a luminance 

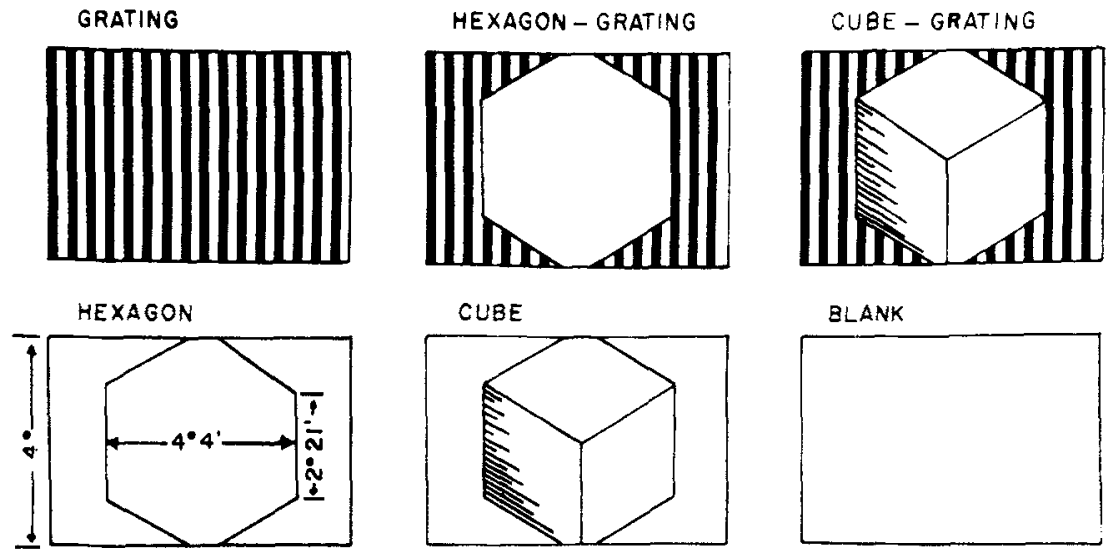

TARGET

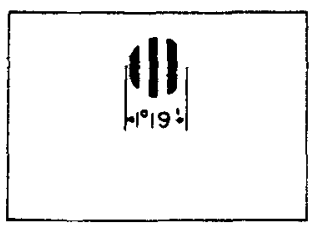

Fig. 1. The stimuli used in this experiment. The first two rows are the adaptation stimuli; $T$ is the test. The dimensions of all cubes and hexagons are as indicated in the hexagon figure in the second row.

of $2 \mathrm{fL}$, so for all fields, mean spatial luminance was held nearly constant (the exceptions being the slight drop in mean spatial luminance due to the lines in the figures which contained a cube or a hexagon). $T$ had the same spatial frequency and contrast as the other grating stimuli and a mean spatial luminance of $5 \mathrm{fL}$.

A trial consisted of an adaptation field, presented for $30 \mathrm{msec}$, followed by $\mathrm{T}$ after one of seven interstimulus intervals (ISI $=0,5,10,20,30,50$, and $100 \mathrm{msec}$ ). Each session was a complete replication of the experiment: for each adaptation condition, one trial at each of the seven ISIs was presented before changing to a new condition. The order of the adaptation fields was randomized per session, and the order of ISIs was randomized for each condition. There were 20 sessions per $S$ and 10 Ss, each with 20-20 vision.

At the beginning of each session, Ss, whose head movements were restrained by a head- and chinrest (Bausch \& Lomb) fixated on a continuously present 4-fL 4-min fixation point in a dark field whose position coincided with the vertex of the $Y$ in the cube conditions. The blank was then presented, followed, after 100 msec, by $T$. The $S$ assigned a modulus of 10 to the apparent contrast of $T$ (the method of magnitude estimations; Stevens, 1966). This constituted the standard. The duration of $T$ was raised or lowered between 12 and 16 msec until the $\mathbf{S}$ felt that a standard clear contrast had been presented. The standard was presented at the beginning of every session and repeated as often as the $S$ desired. Presentation was monoptic. All luminances were measured by an SEI photometer; luminance and duration of all stimuli were subsequently monitored by a Tektronix 504 oscilloscope which displayed the output of the phototubes placed in each channel of the tachistoscope.

\section{RESULTS}

Since magnitude estimations tend to be distributed log normally (Stevens, 1966), all measures and tests were performed on the logarithms of the data. Geometric means were computed for the $10 \mathrm{Ss}$, and the grand mean as a function of ISI for each condition is plotted in Fig. 2. It can be seen that through the first six ISIs, while the differences between conditions are small, the conditions have a generally consistent rank order. From greatest to least adaptation, this order is: grating, cube grating, hexagon grating, blank, hexagon, and cube. The rank orders change at ISI $=100$, although this does not change the mean rank order across all ISIs.

A repeated-measures ANOVA, with the error term being the next order interaction involving $\mathrm{Ss}$, showed all main and interaction effects (except those involving $\mathrm{Ss}$, which cannot be tested in this design) to be significant at least at $p<.01$. This result is not due simply to the contribution of the continuous grating, since the same design ANOVA run on all conditions except the grating also showed all testable main and interaction effects to be significant at $p<.01$. A Duncan's new multiple-range test compared each mean with each other. ${ }^{1}$ All conditions differed from the grating at $p<.05$; the cube-grating differed from the cube at $p<.05$. Otherwise, no condition differed significantly from any other.

Aside from the grating, then, the only other two conditions which differ significantly from each other are the cube-grating and the cube. Thus, depth cues appear to be important in producing significant differences between a figure which appears on a blank background and a figure which appears against a grating background. But failure to reject the null hypothesis for the other conditions (in particular, for the hexagon/hexagongrating difference) cannot be taken as equivalent to the statement that no differential adaptation effects were present in these cases. It is clear from Fig. 2 that adding an outline figure to an otherwise blank field lessens adaptation somewhat and that adding a grating to the outside of the figure deepens adaptation somewhat, whether or not that figure contains depth cues. Moreover, the difference between hexagon-grating and cube-grating adaptation was not significant, and thus the failure to find significant differences between hexagon and hexagon-grating adaptation might simply be a Type II error.

In general, Type II errors are likely in considering the data of this experiment for the following reasons: All effects were small; overall differences between means were generally no larger than 1 . These small

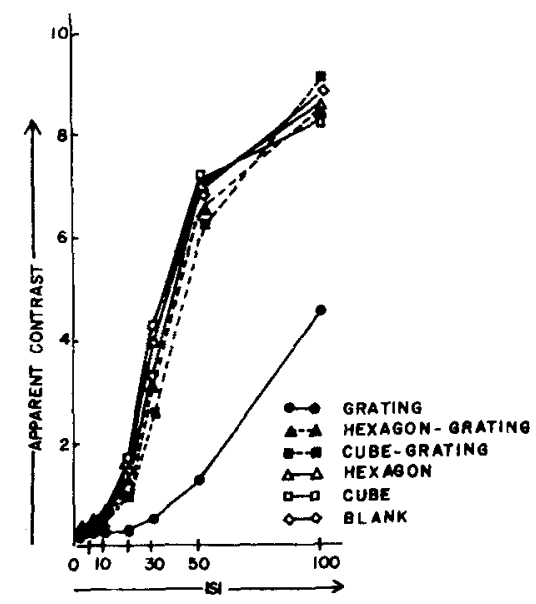

Fig. 2. Apparent contrast of $\mathrm{T}$ as a function of interstisaulus interval for the six adaptation conditions. 
differences (compare with Weisstein, $1970 \mathrm{a}$, where the difference between cube and cube-grating goes as large as 4) may be due to three factors. First, the mean spatial luminance and duration was high; the blank field alone produced a large effect. This large effect may have obscured somewhat the smaller patterned-field masking differences. In addition, the spatial frequency of the grating portions of the stimuli was quite low, and this no doubt reduced the effect somew hat [e.g., see Blakemore \& Campbell (1969), where all adapting frequencies lower than $3 \mathrm{c} / \mathrm{deg}$ produce a maximum adapting effect at $3 \mathrm{c} / \mathrm{deg}$ ]. Finally, as Fig. 2 shows, conditions cross over at ISI $=100$, and this reduces the differences between overall means. It is not clear why this crossover occurred. The blank itself dropped from an estimation of 10 as pretest modulus to a mean of about 9 . While this may be explained as a buildup of light adaptation effects through successive trials, such an explanation would not account for the failure of other conditions to converge to this value at ISI $=100$. Moreover the variation at ISI $=100$ is not due to the magnitude estimation measure, since forced-choice responses on a similar task (Weisstein, 1972a) also show the same kinds and magnitudes of crossovers at ISI $=100$. Thus, it is not at all clear why conditions change their rank order at ISI $=100$; it may have something to do with differing temporal characteristics of different subsets of neural populations. In any case, these crossovers reduced the magnitude of the overall effects.

Given, then, that the overall estimate of the differences between means may be compressed (due either to the experimental design or to crossovers at ISI $=100$, which may represent real differences in temporal recovery functions), there is some reason to investigate the rank orders of the conditions further, using a measure which would be more sensitive to the possible differences between conditions.

One way of probing the small differences between conditions is to compare means without using the data at ISI $=100$. This is, of course, an ad hoc procedure, since it is not clear why the crossovers occur at ISI $=100$; however, adaptation functions in general are known to differ in recovery rates (Matthews, 1971), and masking functions in general have different shapes. Taking, in effect, a "window" over a certain ISI range may therefore have some justification.

Another Duncan's test compared each mean with every other mean across all ISIs except $100 \mathrm{msec}$. The following additional significances and changes in significance were tound. The cube grating differed from the cube and the hexagon at $p<.01$; the cube differed from the blank at $\mathrm{p}<.05$. Finally, although hexagongrating vs cube-grating are within a subset which do not differ significantly, a comparison on these two means alone (correcting the error term by multiplying it by $\sqrt{2}$, as is the procedure for orthogonal comparisons) shows greater cubegrating adaptation at $\mathrm{p}<.05$ (two-tailed). [If, on the other hand, a priori (orthogonal) comparisons are done on cube/cube-grating and hexagon/hexagon-grating, the former is significant at $\mathrm{p}<.01$ and the latter is still not significant.]

Both ad hoc and conservative ways of testing the data show that cube-grating adaptation differs significantly from adaptation to a cube on a blank field but that hexagon-grating adaptation does not differ significantly from adaptation to a hexagon on a blank field. Thus, depth cues appear to be important to the strength of the differential adaptation effect. Ad hoc tests of the data (consideration of ISIs 0 through 50) show, in addition, that the hexagon-grating and cube-grating directly differ from each other.

These data do not lend themselves easily to explanations based either on simple spatial spread (Step a mechanisms) or on eye movements. Both of these explanations would predict as much adaptation for the hexagon-grating as for the cubegrating. In another study (Weisstein, $1972 \mathrm{a}$ ), it is shown that the effect for cube-gratings is orientation-specific and that the apparent contrast of $T$ does not decrease if cube- or hexagongratings are presented for only $160 \mathrm{msec}$. This latter finding casts additional doubt on an explanation for either cube- or hexagon-grating adaptation which assumes simple Step a logic, since effects attributed to this logic occur for stimuli of very short duration (e.g., $25 \mathrm{msec}$; Weisstein, 1972b).

As mentioned, differential eye movements cannot be ruled out until directly tested; nevertheless, as also mentioned, this explanation does not seem a strong candidate given that the $Y$ of the cube and the fixation point coincide, and this would seem to focus attention at the fixation point; and given the angular separations between grating portions of the stimuli and the perimeter of $\mathrm{T}$.

It has been suggested that differences in mean luminance may account for the effects obtained. As mentioned, corrections were not made for the small reduction in mean luminance due to the lines in the hexagon and cube stimuli. But, although an explanation based on differences in mean luminance might account for a decrease in adaptation effects for the nongrating conditions, namely, the blank, hexagon, and cube in that order, it would also then predict that the cube-grating would produce less adaptation than the hexagon grating, whereas the reverse was true. In fact, if mean luminance explains enhancement of the cube relative to the blank, then the amount of adaptation of the cube grating may be underestimated, since attenuation of adaptation would be expected for the cube-grating as well. Thus, differences in mean luminance do not predict the rank order of conditions, nor do they predict the significant differences obtained.

Finally, it should be noted that, while not statistically significant, the hexagon-grating does show greater adaptation than the hexagon, cube, or blank. It may be that the hexagon itself, a bounded contour, sometimes looks like it is in front of the grating, or it may be that a number of mechanisms contribute to the interrupted-grating adaptation effect. For instance, it is possible that both hexagon- and cube-grating adaptation indicate the activity of neural populations which are coding for frequency; one would theorize that such coding should not be location-specific. (Frequency coding itself may be synergistic with neural representation of depth: upon pilot testing control interrupted-grating stimuli, it was extremely difficult to construct a perspective drawing of a figure which clearly looked as if it were behind the grating.) In any case, in addition to frequency coding, one would still have to explain the role of depth cues in the strength of the adaptation effect.

In summary, these results show differences in hexagon-grating and cube-grating adaptation. At very minimum, these differences indicate that simple spatial spread and/or eye movements are not adequate to explain all of the results. Thus, depth cues appear to be important to the strength of interrupted-grating adaptation, and this may imply that the effect does indeed have something to do with neural mechanisms which separate a scene into its components.

\section{REFERENCES}

BL A KEM ORE, C., \& CAMPBELL, F. W. On the existence of neurones in the human visual system selectively sensitive to the orientation and size of retinal images. Journal of Physiology, 1969, 203, 237-260

GUZMAN, A. Object recognition: Discovering the parallelepipeds in a visual scene. Proceedings of the 2nd Hawaii 
international Conference on Systems Sciences, 1969, 479-482.

KIRK, R. E. Experimental design procedures for the behavioral sciences. Monterey, Calif: Brooks/Cole, 1968.

MATTHEWS, M. Spatial and tem poral factors in masking by edges and disks. Perception \& Psychophysics, 1971, 9, 15-22.

SEKULER, R., \& ARMSTRONG, L. R. Technical comment on neural symbolic activity. Science, $1970,170,1226-1227$

STEINMAN, R. M. Effect of target size,

luminance, and color on monocular fixation. Joumal of the Optical Society of America, $1965,55,1158-1165$.

STEVENS, S. S. Duration, luminance, and the brightness exponent. Perception \& Psychophysics, 1966, 1, 96-100.

WEISSTEIN, N. Neural symbolic activity: A psychophysical measure. Science, $1970 \mathrm{a}$ 168, $1489-1499$

WEISSTEIN, N. Reply to Sekuler and Armstrong. Science, 1970b, 170 $1227-1228$.

WEISSTEIN, N. Orientation-specific cube-grating adaptation and absence of short-duration effects. Joumal of the Optical Society of America, 1972a, in press. (Abstract)

WEISSTEIN, N. "Metacontrast." In D. Jameson and L. Hurvich (Eds.), Visual psychophysics. Heidelberg: Springer, $1972 \mathrm{~b}$.
NOTE

1. Although the data were transformed into logarithms, variances for each condition were still not homogeneous laverage $F(9.9)$ $>10, \mathrm{p}<.011$. Therefore, in the $\mathrm{q}$ statistic computed for reference to Duncan's tables (Kink, 1968), an exor term was computed individually for each comparison between means (rather than taking the overall estimate of error from the Subject by Conditions interaction term of the ANOVA). This error term was $\left(S_{d_{i j} / n}\right)^{1 / 2}$, where $S_{\mathrm{d}_{\mathrm{j} j}}=$ the variance of the difference between means paired by $S$ s on Conditions $i$ and $\mathrm{j}$. 